\title{
Diagnosis of Finger Dysfunction Caused by Ulnar Nerve Lesion
}

\author{
Jurgen Rahmel, Christian Blum \\ University of Kaiserslautern \\ Centre for Learning Systems and Applications \\ P.O. Box 3049, 67653 Kaiserslautern, Germany \\ e-mail: rahmel@informatik.uni-kl.de \\ Peter Hahn \\ Neustadt Hand Centre \\ Salzburger Leite 1 \\ 97616 Bad Neustadt, Germany \\ e-mail: hahn@hand.franken.de
}

\begin{abstract}
In this paper we introduce a novel approach for diagnosis and monitoring of ulnar nerve lesions, affecting the coordination of movement of the ring and little finger of the human hand. Symptoms to be observed are static and dynamic anomalies in movement pattern. Based on data generated by ultrasound measurements, we developed suitable preprocessing methods for automatic extraction of relevant features from the movement pattern to be examined. The partial absence of class information even for the patterns in the training set requires the use of unsupervised methods for the learning and class assignment procedures, since common classification or decision tree methods are not applicable. For that reason, we use a new dynamic and hierarchic neural network for the analysis of the generated pattern vectors. The property of topology preservation as well as the dynamically structured architecture of the network make it suited for the special needs of this medical task, such as providing variable levels of generalization and
\end{abstract}


efficient retrieval of similar cases for diagnosis purposes. We present a visualization of the clustering structure and classification results.

Keywords: Neural Networks, Topology Preservation, Hierarchical Clustering, Medical Diagnosis, Motion Analysis, Data Visualization

\section{Introduction}

Peripheral nerve injuries are common and frequently disabling. Functional recovery following nerve repair in upper extremity and hand injuries is often the rate-limiting factor in determining ultimate hand function. Besides external injuries all nerves can be damaged by entrapment neuropathies where nerves are injured due to compression in anatomical bottlenecks.

The human hand is provided with the radial, median and ulnar nerve. The ulnar nerve provides sensory function for the small and ring finger and innervates the intrinsic muscles of the hand. These muscles are crucial in balancing and coordinating the flexor and extensor muscles, rendering possible fine movement such as grip and pinch.

While assessing sensory function is easy, objective analysis of motor function is quite difficult. Clinical investigation includes grip force measurement and recording of active and passive range of motion. Beside these factors ulnar nerve dysfunction causes changes in coordination of the movement which cannot be measured by instruments. In contrast to a normal, physiological movement pattern (Figure 1(a)), the dynamic disorder 'rolling' describes the pathological flexion of the finger. This movement resembles the rolling of a carpet (figure 1(b)). As a result, patients are not able to grasp an object because their fingers push it out of the palm. The static disorder 'clawing' describes the hyperextension of the MP joint with 


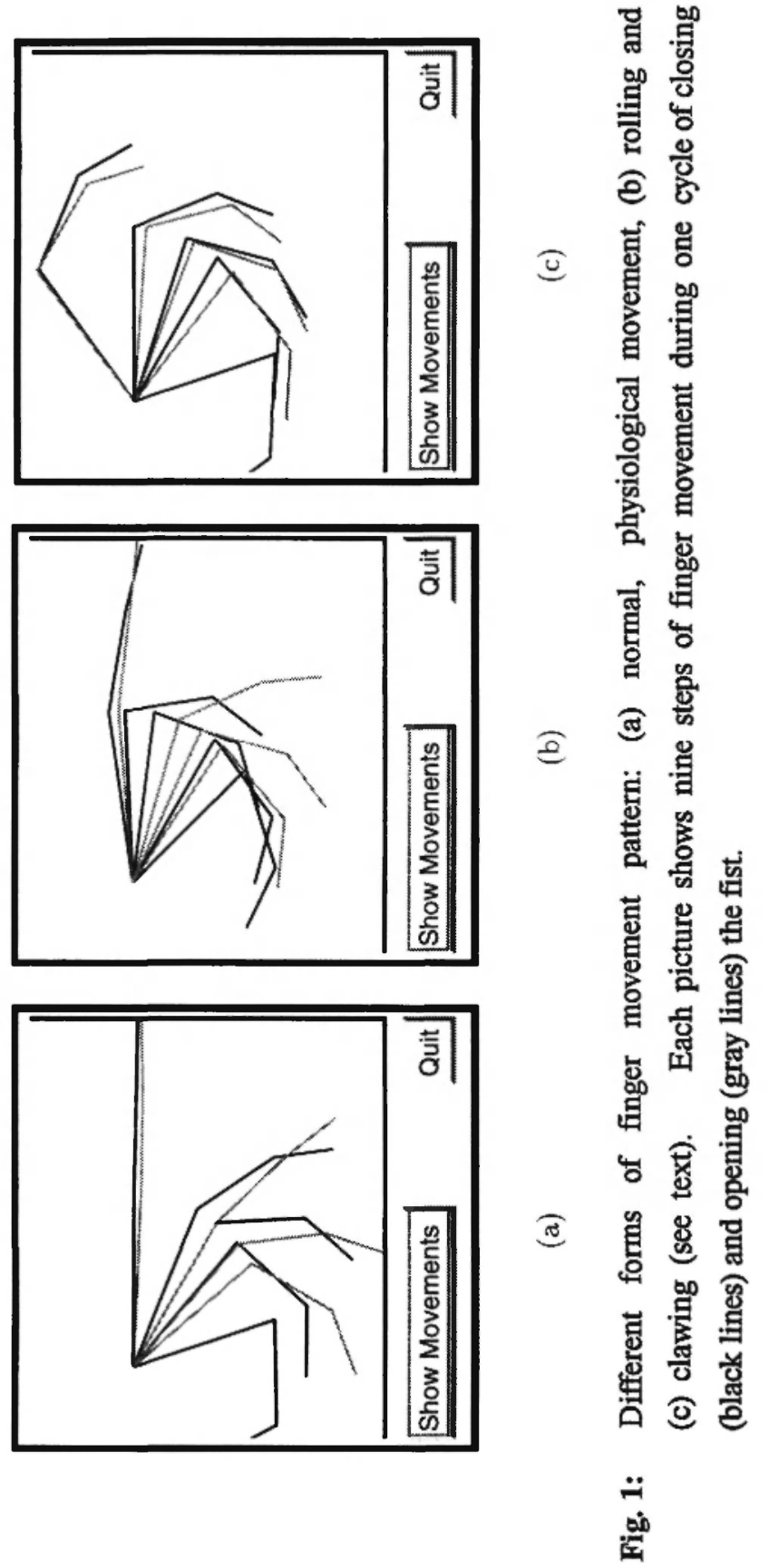


flexion of the PIP and DIP joint ${ }^{1}$ while the finger is in resting position (figure 1(c)). These descriptions are based on the experience of the examiner. Changes in quality and especially improvement of fine motor activities after nerve repair are difficult to quantify. If nerve repair fails, there are different operations to rebuild the movement pattern (e.g. the Zancolli-lasso-plasty mentioned in section 5). In these cases, the success of surgery also cannot be quantified. Until now, there was no convenient measurement system to distinguish finger movement patterns.

Based on kinematic research we established a measurement system to get real-time data of human finger movement. Classical mathematical methods like discriminant analysis to analyze the data in order to distinguish between normal and pathological movement failed. Statistical clustering provides a good first insight in the structuring of the data but is not able to support the specific needs in this application (cf. section 2), e.g. the utilization of neighborhood relationships between clusters for retrieval and comparison aspects. Other decision methods like CART [Breiman et al., 1984] or decision trees [Quinlan, 1993] have some desired diagnostic properties but are not applicable because of missing class information in the data records of our domain. Another crucial point is the fact that the prospective diagnosis system should efficiently support the medical experts in decision making. Thus, for any given data vector, the system has to be able to retrieve a number (that is possibly not known exactly in advance) of similar stored vectors in order to provide an overview of the current situation.

A particular class of neural network models has the potential to solve this problem. Regarding the mapping from the input space onto the space spanned by the neighborhood relations of the neurons in the network, the property of certain neural network models to keep track of neighborhood relationships of clusters of data even in cases of reduction of dimensionality is called topology preservation. The degree of topology preservation can be determined by the observation of how well neighborhood relationships in one space are preserved by the mapping onto the other space. Thus, one

${ }^{1}$ The MP, PIP and DIP joints are the three finger joints ordered from the base joint between hand and finger to the tip. 
question is: for two input vectors that are close in input space, are their best matching units close ${ }^{2}$ in the network topology? The other question is: for two neurons that are neighbors in the network topology, are their associated weight vectors close in the input space? The SplitNet model used in our approach combines the retrieval advantages of topology preserving nets with hierarchical structuring of data usually known from classification or decision trees only.

In section 2, we outline some domain specific problems that determine some of the required properties of a suitable analysis method. Section 3 describes some commonly used methods in the field of ulnar nerve examination and previously used diagnosis approaches. We then briefly introduce in section 4 the neural model that is the basis of our approach. We demonstrate the results of the network application in section 5 and conclude the paper with a summary.

\section{Domain-Specific Medical Requirements}

In this section, we will describe some of the characteristic problems in our domain of ulnar nerve diagnosis and outline the main medical goals to be aimed at with current and future research.

The first problem for data analysis in this domain is the visualization and explanation of data structure to the human observer. We found a hierarchical organization to be computationally tractable as well as easily providing access to useful information on the data structure and various levels of abstraction. Some conventional hierarchical methods are reviewed in section 3 while our approach with a new hierarchical neural network is described in section 4.2 .

A particular characteristic of our domain is the (at least) partial absence of class information. From the patient records we only know if a patient had

${ }^{2}$ As different input vectors may be mapped onto the same neuron, in this informal explanation, the closeness of neurons includes also the identity of those neurons. 
a nerve lesion or not, but this knowledge is not relevant for diagnosis. Fully recovered finger motion should be considered to be healthy, regardless of the former presence of a lesion. Thus, diagnostic information has to be supplied by the experienced medical expert. Since visual inspection of finger movement can only reveal strongly developed symptoms and provides only a subjective judgement, we first had to develop measurement and preprocessing methods for objective quantification of movement pattern. Section 4.1 gives details on this approach. The generated patterns always have to be related to similar cases in order to be interpretable by the medical expert. We use the $k$-nearest-neighbor rule for the judgement of a new pattern, i.e. in the most similar of the trained patterns will be consulted in order to estimate the kind and degree of dysfunction represented by the new feature vector. The analysis method therefore has to support the retrieval of similar cases, at best in an incremental way, because the number of patterns for comparison is usually not known a priori and depends on the matching of nearest neighbors with the pattern that is to be evaluated. The generation of appropriate memory and indexing structures is one of the crucial points for application development.

This indexing structure should be the basis of the method that is able to detect less severe forms of motion dysfunctions. Only very pronounced changes in static and especially dynamic anomalies are accessible by visual inspection. They mostly appear after serious lesions of the nerve, yet do not necessarily appear or persist after lesions. Less severe forms (e.g. caused by slowly progressing entrapment neuropathies) as well as possibly not yet discovered kinds of motion disturbances should be detectable and quantifiable. A similar problem arises for the task of monitoring motion recovery success after healing of the nerve or surgical reconstruction of motoric function of the finger. Recovery after nerve repair depends on several factors, e.g. age of the patient, level of injury, and delay of repair. Besides these predictable factors, individual biology plays an important role especially in motoric recovery. Thus the results are not predictable, but should be made comparable. 


\section{Related Approaches and Methods}

This section outlines the need for a new approach to ulnar nerve diagnostics and summarizes some of the well known methods for pattern analysis that normally are applicable for related tasks.

\subsection{Previous Attempts to Ulnar Nerve Examination and Diagnosis}

Traditionally, monitoring included two different aims. Global hand function can be assessed by measurement of grip and pinch strength, active ( $\mathrm{TAM}=$ total active motion) and passive (TPM = total passive motion) range of motion and description of individual tendon function. Specific lack of hand or finger function is described by different clinical signs. These signs give a pictorial description of disturbed function as e.g. rolling or clawing. These signs depend on subjective impressions of the examiner so they so far could not be used for quantification of dysfunctions. [Srinivasan, 1983] for the first time tried to establish a system to assess motor function using a graphical representation of defined finger positions. More or less this system defines a working space but is not able to evaluate fine changes in dynamic finger function. Electrophysiological investigations such as neurography and myography describe the degree of nerve or muscle recovery, but they do not correlate directly with functional outcome.

\subsection{Pattern Recognition Methods for Classification and Structuring}

In this section, we give a brief review of some basic methods usually applicable in a pattern analysis task. We outline the fundamental properties and mention insufficiencies for our domain. Since we are interested in structuring the data set we focus our discussion on hierarchical methods.

\section{Hierarchical Cluster Analysis}

The hierarchical cluster analysis, either the divisive or the agglomerative methods, are methods that progressively split or link clusters of data. The 
result of the method can be visualized as a dendrogram, which is a twodimensional tree structure that shows the order of linkage (for the agglomerative case) and the distance or similarity at which this linkage of clusters was performed. Thus, this method is able to display the clustering of data but since it provides no topological information on the neighborhood relations between clusters other than the linkage, it is not possible to reason about the real spatial relationship of the observed pattern or use the structure for retrieval purposes. There is no similarity information other than the one for linked clusters. Similar statements are true of course for divisive methods. So the hierarchical clustering methods are useful tools for a preliminary analysis of the data, but they do not provide additional ways for explanation of the clustering results and do not enable reasoning on alternative solutions based on neighborhood observations.

\section{Tree-structures for classification}

The classification trees [Breiman et al., 1984] are a tree-structured representation of a classifier consisting of decision nodes, the nonterminal nodes in the tree, and class assignment nodes, the terminal nodes. In the decision nodes, the solution space is separated into two subspaces by testing a linear combination of attributes against a threshold. Thus, on a path through decision nodes on a branch of the tree, a sequence of hyperplanes will classify a given input vector into the subspace belonging to one of the terminal nodes. The orientation of the hyperplanes is determined separately for each decision node and chosen to optimize an a posteriori criterion like the misclassification cost. This computationally expensive strategy is looking for an optimal division of the current training set but may not be adaptable for incremental learning tasks. Additionally, like for the clustering methods, the topological information of neighboring subspaces is lost during the sequential splitting by hyperplanes.

Decision trees, e.g. [Quinlan, 1986] and the dynamic version C4.5 [Quinlan, 1993], are symbolic learning methods that construct a tree structure by, e.g., considering an information gain criterion. According to 
this criterion, at each level of the subtrees, an attribute and a partition value are selected, in order to form a decision at this branch. This method yields trees with the shortest average path length (i.e. the number of decisions necessary to assign a class) but as in the other methods described above, the successive separation of the input space by orthogonal hyperplanes destroys neighborhood relationships between the leaves of the tree, a fact that becomes important in application with missing, uncertain or fuzzy (numeric) data.

There exist a number of neural network models that are called treestructured and which, considering the network topology of nodes and propagating links, are indeed trees. The construction of these neural trees, however, is not done in a hierarchical way by a neural training mechanism. Tree-structured MLP networks like the Perceptron Trees [Utgoff, 1988] are quite comparable to decision trees (see below) as they use an attribute test at each decision node. But unlike in decision trees, now the terminal nodes are not class assignment nodes, but Linear Threshold Units (LTU), which divide the current subspace by a hyperplane. Thus, the terminal nodes require an additional test and then provide a class assignment. A classification along a path in the Perceptron Tree corresponds to a division of the solution space by hyperplanes perpendicular to the attribute dimensions and only for the last test contained in the terminal node, arbitrary orientation of the decision plane is allowed.

A model based on a competitive tree structured network is proposed in Fang et al., 1991], [Li et al., 1992]. Those binary Neural Trees have a predefined size and structure and may be trained with variants of the competitive learning scheme. Input data is forwarded through the tree and at each level it takes the path with the winning neuron. The weight adaptation affects the complete subtree of the first winning neuron. Thus, again we have a subsequential division of the data space by hyperplanes, but for the Neural Trees, the orientation of the planes may change during weight adaptation. The classification structure is more flexible than e.g. the one for classification or decision trees, but the need to predefine the network structure is a drawback. 


\section{A New Approach to Ulnar Nerve Diagnostics}

In this section, we briefly introduce the methods we use for the task of analyzing finger movement pattern. Details on patient data and interpretation of training results follow in section 5 .

\subsection{Data Generation and Preprocessing}

We used a real time motion analysis system based on ultrasound (CMS50, Zebris, Germany). Principles of this system are described in [Guggenbuhl \& Krilger, 1987]. Fixing eight markers on the dorsum of the metacarpal bones and on each phalanx of the ring finger (paired by two, in order to represent the direction of the bone), we obtained the three dimensional coordinates of each marker. Each marker emits 25 pulses per second. From the coordinates we calculated the angles in the twodimensional area of movement during opening and closing of the fist. The technique of measurement was tested by fixing the markers on a standard finger goniometer. These control measurements showed that angle measurement is reliable and is not influenced by translation or rotation of the limb. Multiple measurements performed on the same person demonstrated the accuracy of the measuring process. The arms of the patients were fixed on a support with the shoulder abducted by 45 degrees. The wrist joint was not fixed to allow normal antagonistic movement of the wrist during extension and flexion of the fingers. Details of the measurement are described in Hahn et al., 1995]. Plots of the data show three time dependent sinusoidal curves (cf. figure 2 for an example). Normally ring and small fingers are affected by ulnar nerve lesion. We used the ring finger for the SplitNet model since the movement of the small finger may alter, even in healthy persons, due to anatomical variations.

Preprocessing of these data was difficult because two different problems had to be solved. First of all, selected sets of data should represent the same view of the original data. Simple methods failed because patients moved with different velocities. Thus these sets were not comparable. On the other 


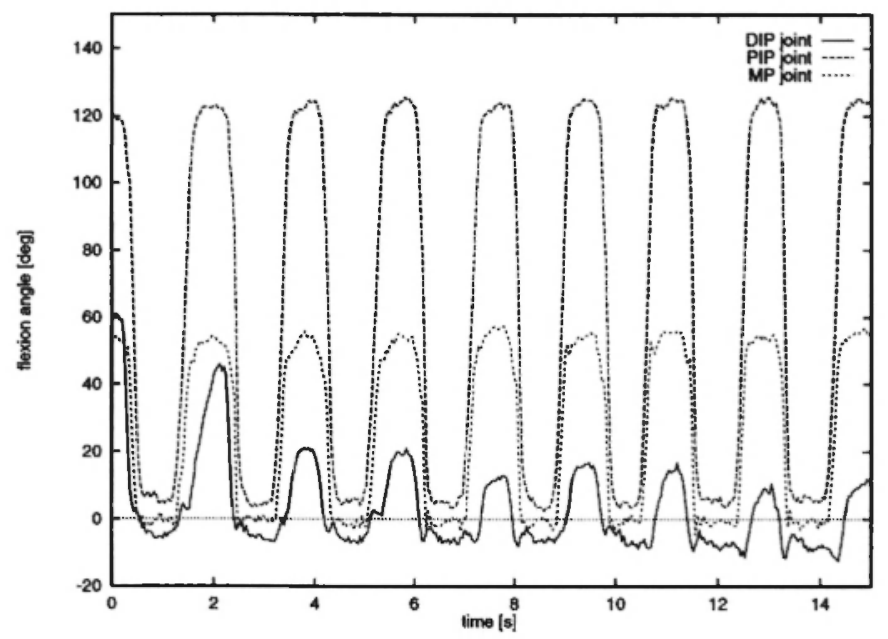

Fig. 2: Plots of the three finger joint angles during fist opening and closing.

hand, selected features and the results of training should include interpretability for medical experts by representing the whole range of movement and enabling a graphical description of different flexion angles. As a result, we implemented a tool to automatically select feature vectors from the measurement data by observing predefined positions of finger movement, i.e. angular position of the finger tip with respect to the dorsum of the hand, during the closure and opening of the fist. This set of data contains the three angles of the respective finger joints for each selected finger position. Using these angles and the lengths of the phalangeal segments as described by Littler \& Thompson, 1987], who defined a length of 2 for the end, 3 for the middle and 5 for the proximal phalanx, we could construct an image of the individual movement pattern (see figure 1 for examples). For the reason of retransformability of training results into interpretable pictures (see figure3), we used no further dimension reducing preprocessing methods such as, e.g., principal component analysis. 


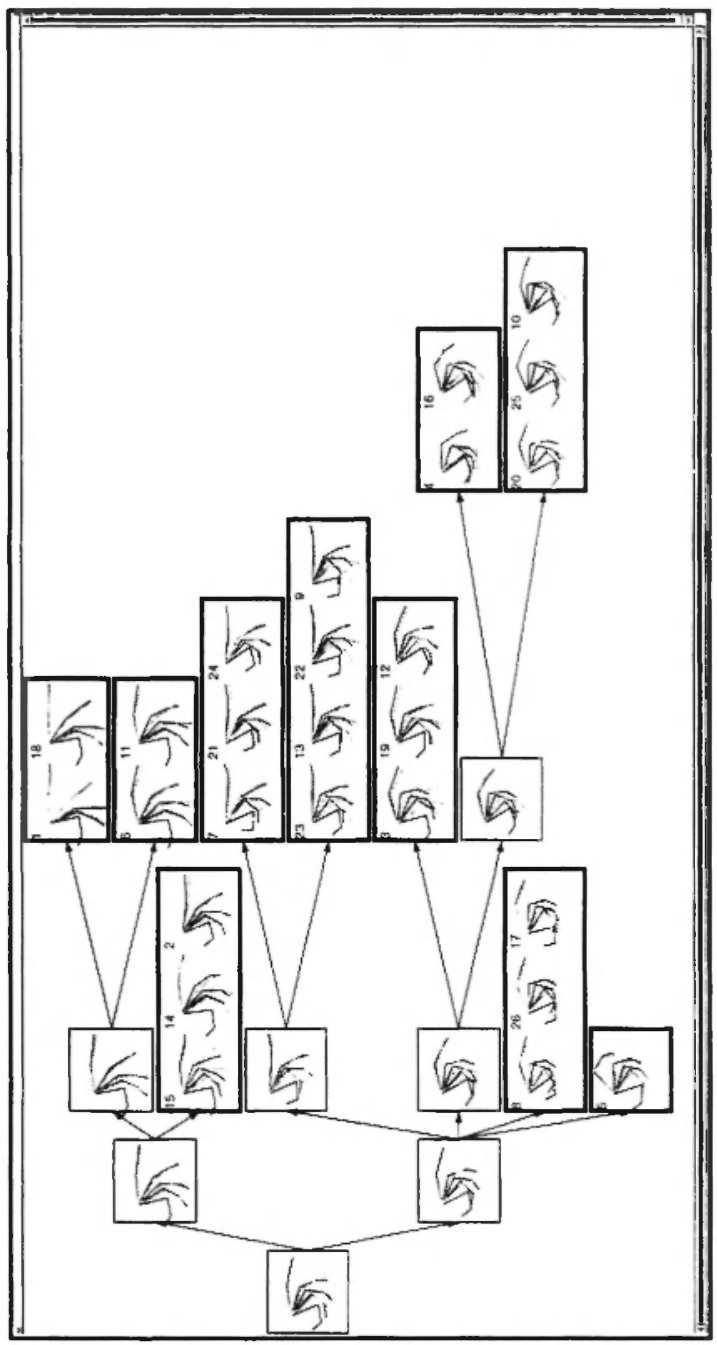

(a)

Fig. 3: Hierarchical representations generated by SplitNet. The retranslation of neuron weights allows the display of interpretable finger movements in the learned hierarchical arrangement (a). The same tree is shown with a text output (b) for naming nodes by their box number (first line in each box) and mapping training vectors onto neurons. The three-letter acronyms denote the patients, patients for neighboring neurons are separated by a semicolon. 


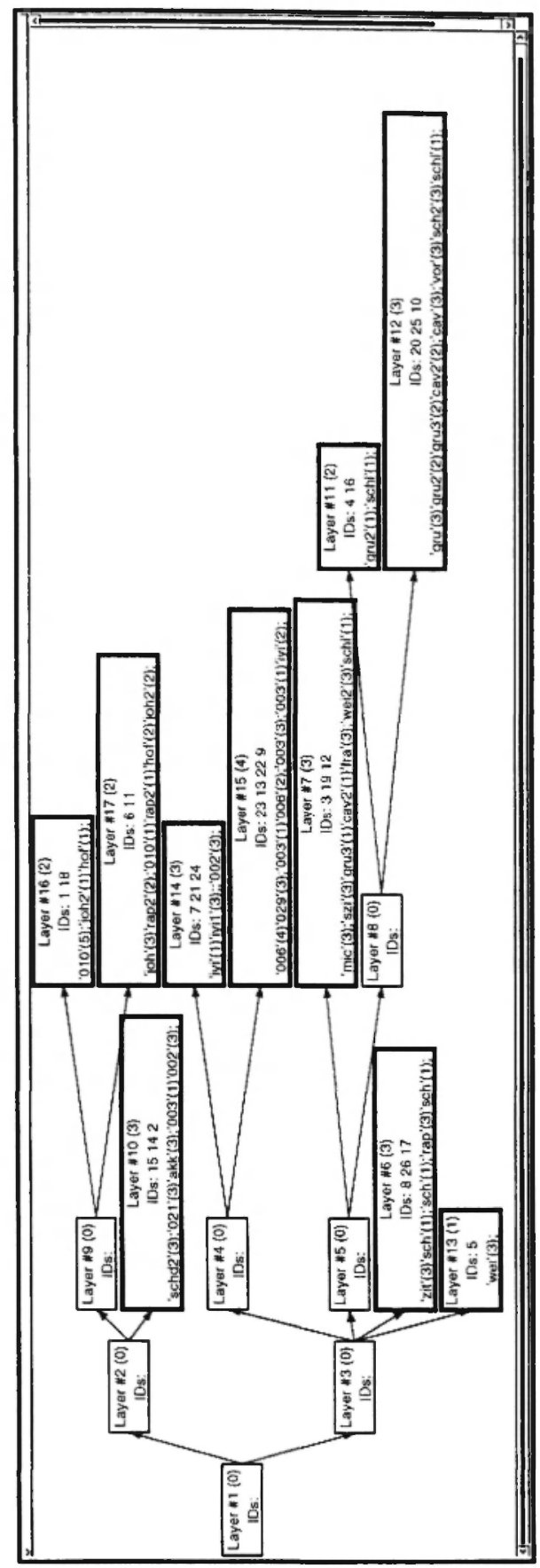

(b) 


\subsection{The SplitNet Model: Hierarchical Structuring of Data}

As indicated in section 3, existing approaches to clustering and classification neglect the spatial relations of clusters or partial solution spaces. A variety of neural network models have the property of being topology preserving and thus have the potential to provide neighborhood information that is essential for the intended $k$-nearest-neighbor approach to diagnosis. As described in the introduction, measuring the topology preservation involves observation of the number and sizes of discontinuities for the mappings from input space into the network topology and vice versa. These questions led to the development of the topographic function Villmann et al., 1996], that effectively quantifies the topology preservation in topographic maps. There also exist other approaches like the topographic product [Bauer \& Pawelzik, 1992] or the topographic error [Kiviluoto, 1996].

SplitNet is a topology preserving, dynamically growing model for unsupervised learning and hierarchical structuring of data [Rahmel, 1996b].. Starting with a single Kohonen chain [Kohonen, 1990], localized insertion and deletion criteria enable an efficient quantization of the data space. The hierarchy in the architecture develops, when one of the following splitting criteria is satisfied: (i) detection of topological defects, (ii) deletion of neurons by an aging mechanism, (iii) significant local variances in quantization errors and (iv) significant local variances in edge lengths. Those criteria are constantly checked during the network training. If a criterion is satisfied, the affected chain is split into two or more subchains that are added to the network at one level lower in the hierarchy. The topology preserving construction of the network structure provides local neighborhood information that is necessary for incremental retrieval of nearest neighbor to a given input vector. A more rigorous and exhaustive treatment of these aspects and retrieval results can be found in Rahmel \& Villmann, 1996]..

Since unsupervised learning methods provide no direct classification, the training result has to be interpreted in the context given by the training data. For the SplitNet model, we observe three containers of information that can 
be used for the tasks in diagnosis applications like the one described in this paper:

Neuron distribution: An insertion criterion based on the minimization of reconstruction error determines the error function to be minimized. Quantization of the data set allows local estimation of density and probability of class occurrences (if they are known for some or all of the training patterns).

Topology: The connections between neighboring neurons provide information on where to find similar cases. Measuring topological defects yields the search depth for incremental retrieval of nearest neighbors to a given query Rahmel, 1996a].. This offers an approach to implement efficient algorithms for the retrieval of similar and possibly relevant, comparable cases.

Hierarchy: The hierarchical structure of the network contains different levels of generalization and abstraction and thus gives an insightful visualization of the data structure for the domain expert. On the other hand, it naturally accelerates the training process, since it allows a fast tree search for best matches.

Among the known topology preserving network models are the Growing Cell Structures (GCS) [Fritzke, 1993] and the Neural Gas algorithm [Martinetz \& Schulten, 1991].. The Neural Gas, as well as its descendant, the Topology Representing Network (TRN) [Martinetz \& Schulten, 1994]., learns by positioning a predefined number of reference vectors according to an error measure and constructing the topological neighborhood connections in the output space from scratch. There is no initial structure like a fixed neuron grid. The GCS on the other hand use elementary simplices of a given dimensionality to grow a network by increasing the number of simplices in the network. The growth process is guided by some error criterion like quantization error.

The main improvement introduced by the SplitNet model is the development of a hierarchically structured, yet topology preserving representation of the data. It integrates two necessary properties formerly not 
provided by a single method. One is the facility to analyze the data at various levels of generalization with the help of expressive visualizations of structure and data contents (cf. section 5). The other is the ability to retrieve related data for diagnostic evaluation of a new data set. The task of comparing a given data vector of a new patient to similar vectors in the network is fully accomplished by the topology-based retrieval algorithms described in [Rahmel \& Villman, 1996]. The utility of the constructed hierarchy is explained in detail in the following section for the domain of ulnar nerve lesions.

However, we have to stress the point that the tree structure itself is not a classifier in the sense of, e.g., a classification tree. The mere fact that two different data vectors are mapped onto the neurons in the same layer, i.e. the same terminal node in the tree, does not necessarily lead to the conclusion that the two vectors represent exactly the same category. This assessment depends additionally on the spatial relationships of neurons and is supported by examination of the local network topology.

\section{Application Results and Interpretation}

\subsection{Patients, data sets and network training}

24 measurements of ring finger movements of 16 patients suffering from different lacerations of the ulnar nerve were obtained. One (gru) was measured before and two times after release of the ulnar nerve at the elbow level. Four patients (wei, rap, joh, sch) were measured before and six patients (wei2, rap2, joh2, sch2, mic, hof) after reconstruction of motoric function by Zancolli lasso plasty. These data were compared to the data of ten fingers of six healthy persons, in the following represented by acronyms composed of digits.

Training data sets were generated by the methods described in section 4. 1. For the results reported here, we used 102 data sets of one close-open cycle, respectively. Figure 3 shows the hierarchical representation of the data as it was generated by SplitNet. The training was automatically 
stopped, when the network developed a size and structure, for which the quantization error distribution over the different clusters was satisfyingly homogeneous, i.e. when the estimated error values where in a range of less than $40 \%$ of the minimum error estimated for any of the leaf nodes in the tree. The trees generated with this stopping criterion were found to be in compliance with the experts requirements of having an accurate, but still not overfitting representation of the data. In total, clinical examination and measurement provided up to now more than $\mathbf{5 0 0}$ data sets and the number is constantly growing as new patients are examined. We used the restricted data set for demonstration purposes; all results are fully transferable to the whole available amount of data.

\subsection{Medical Interpretation of Tree Content}

For evaluating the results of the application of the SplitNet model to clinical data, it is necessary to compare clustering and distribution of data generated by the model with the facts known by medical experts. Only if the interpretation, as it is proposed by the tree structure, matches the clinical knowledge available for these patients, we can argue that SplitNet is a useful tool for generating a 'data base net' that may be used for interpretation and diagnosis of new data for which the clinical history is possibly unknown.

SplitNet early separates at the first level into branches which are numbered \#2 and \#3 in the text version of Figure 2. Depending on layer \#3 two branches develop. The branch depending on layer \#4 contains mainly normal movements. In fact one patient (iyi) is included in these layers, but, although this subject suffers from ulnar nerve laceration, the finger movement appears to be normal. Thus, this placement of the patient in the tree structure is in accordance with clinical investigation.

The lower part of branch \#3 containing \#5, 6 and 13 present different kinds of ulnar nerve palsy. Layer \#6, 11, 12 and 13 present combined 'rolling' and 'clawing' caused by dynamic and static disturbance of movement pattern. For patients in layer \#6 and 13 the interphalangeal joints are more fixed than in layer \#11 and 12. Layer \#7 shows 'clawing' without 'rolling'. From a clinical point of view all patients suffering from severe 
ulnar nerve palsy can be found in leaves \#6, 7,11, 12,13. Dividing the patients according to the severity of movement disturbance, \#6 and 13 seem to be most and \#7 least disabling.

Layers \#16 and 17 are of special interest. These layers are separated from the group with ulnar nerve palsy and are topologically connected to the layers containing normal pattern, but the represented patients show a special kind of movement. Flexion is initiated by nearly exclusive MP-flexion followed by late PIP- and DIP-flexion. These two layers contain four of six patients operated by Zancolli-lasso-plasty, an operation to restore normal finger function by transposing of a flexor tendon. As evident from our results, finger function is enhanced (as proposed by the neighborhood to normal function), but normal movement pattern could not be achieved. Other tendon transfers and their influence on movement pattern will be tested in future.

Visualization of movement data by SplitNet mirrors clinical reality. Changes in biomechanics caused by nerve laceration and reparation by tendon transfer can be interpreted. At the moment three main areas of movement pattern can be distinguished: normal, pathological and movement pattern after Zancolli lasso-plasty. However, we again state that the hierarchical structure is not the only source of information in the network and its 2-dimensional visualization is possibly not able to represent all aspects of a high dimensional data space. For diagnostic purposes, the automatic search routines therefore refer also to the topological structure of the network. For a given query, the topology search retrieves all those neurons, which belong to the cluster of the best match. This search is localized and thus very efficient.

The question remains, how relevant the retrieved neurons are. First tests with a 'data base net' showed promising results. We trained $70 \%$ of the available data and used the remaining $30 \%$ as a test set. For each test vector, the cluster of the best match was determined and checked if the majority vote of the training vectors represented by those neurons was in agreement with the clinical characteristics of the test vector. With this additional retrieval and test step, the neural clustering model can be regarded as a 
classifier and we obtained a classification accuracy of $92 \%$ for the data available to date. The misclassifications were mostly due to motion anomalies caused by single finger movements differing from the usual personal characteristic of a patient. This is not a technical measurement problem but rather a general difficulty in repeated motion analysis tasks. We are investigating variants of the measurement setup to alleviate this problem.

\section{Conclusion}

Our system for the first time applies pattern recognition by a neural net approach to human finger movement. As we can see from the results, SplitNet is able to decide between normal and different forms of pathological movements. Beside these simple clustering tasks SplitNet provides support for the interpretation of the learning processes which have occurred. Thus, interpretation of the images (which are retranslations of neuron weights into the semantics of training vectors) enhances our knowledge of the movement pattern. For example we recognized that most of the Zancolli-lasso-plasty are separated, because they represent a special type of movement pattern, which resembles intrinsic-plus movement. In a similar way, other types of dynamic or static reconstructions can be tested in the future. At the moment, we still have data of only a rather small number of patients. So we do not know if we portray the whole spectrum of ulnar nerve dysfunction. More data have to be recorded to decide how many different types of changes in movement pattern there are. Data from nontraumatic lesions such as compressions neuropathies have to be gathered. The aim is to build up a neural net containing all types of normal and pathological movement. Then we will be able to represent all ulnar nerve lesions by recording finger movement and classify the new movement pattern by observing the mapping performed by the neural net onto a certain location in the tree, for which clinical diagnosis is already accessible. 
The aim of gaining more data for the different pathological forms of the ulnar nerve dysfunctions also yields a need for incremental learning of new patterns without relearning the old ones. This is part of current research, where we try to use a structured case memory for training. Each training vector is associated with its best matching neuron in the network. Newly generated pattern are trained together with the topologically adjacent located older pattern, a form of training that is enabled by the construction of a network topology as it is achieved by the SplitNet model.

\section{References}

Bauer, H.-U., Pawelzik, K.R. Quantifying the neighborhood preservation of self-organizing feature maps. IEEE. Transactions on Neural Networks, 3(4), 570-579 (1992).

Breiman, L., Friedman, J.H., Olsen, R.A., Stone, C.J. Classification and Regression Trees, Belmont, CA: Wadsworth, 1984.

Fang, L., Jennings, A., Wen, W.X., Li, K., Li, T. Unsupervised learning for neural trees, in: Proceedings of the IJCNN, 1991.

Fritzke, B. Growing cell structures - A self-organizing network for unsupervised and supervised learning. Tech. Rept. TR-93-026, ICSI, 1993.

Guggenbühl, U., Krüger, $H$. Bewegungsanalyse an verschiedenen industriellen Arbeitsplătzen. Sozial-und Proventivmedizin, 32, 266-268 (1987).

Hahn, P., Krimmer, H., Hradetzky, A., Lanz, U. Quantitative analysis of the linkage between the interphalangeal joints of the index finger. In vivo study. Journal of Hand Surgery, 20B, 696-699 (1995).

Kiviluoto, K. Topology preservation in self-organizing maps, in: Proceedings of the ICNN, 1996; pp. 294-299.

Kohonen, T. The self-organizing map, Proceedings of the IEEE, 78(9), 1464-1480 (1990).

Li, T., Fang, L., Jennings, A. structurally adaptive self-organizing neural trees, in: Proceedings of the ICNN, 1992. 
Littler, J.W., thompson, J.S. Surgical and functional anatomy, in: Bowers, W.H. (ed.), The Interphalangeal Joints, Edinburgh: Churchill Livingstone, 1987; pp. 14-20.

Martinetz, T., Schulten, K. A neural-gas network learns topologies, in: Kohonen, T., Mäkisara, K., Simula, O., Kangas, J. (eds.), Artificial Neural Networks. Elsevier Science Publishers, 1991; pp. 397-402.

Martinetz, T., Schulten, K. Topology representing networks. Neural Networks, 7 (2), (1994).

Quinlan, J.R. Induction of decision trees. Machine Learning, 1 (1), 81-106 (1986).

Quinlan, J.R. C4.5: Programs for Machine Learning, Morgan Kaufman, 1993.

Rahmel, J. On the role of toplogy for neural network interpretation, in: Wahlster, W. (ed.), Proc. of the ECAI, 1996a.

Rahmel, J. SplitNet: Learning of hierarchical Kohonen chains, in: Proc. of the ICNN '96, 1996b.

Rahmel, J., Villmann, T. Interpreting topology preserving networks. Tech. Rept. LSA-96-01E, University of Kaiserslautern, 1996.

Srinivasan, $H$. Universe of finger postures and finger dynamography. Handchir. Mikrochir. Plastische Chirurgie, 15, 3-6 (1983).

Utgoff, P. Perceptron trees: A case study in hybrid concept representations, Proc. Nat. Conf. on AI, 1988; pp. 601-606.

Villmann, T., Der, R., Herrmann, M., Martinetz, T. Topology preservation in self-organizing feature maps: Exact definition and measurement. IEEE Transactions on Neural Networks [to appear]. 
